\title{
The Fast Evolution of SN 2010bh associated with GRB 100316D
}

\author{
Felipe Olivares E. ${ }^{1}$, Jochen Greiner ${ }^{1}$, Patricia Schady ${ }^{1}$, Arne Rau ${ }^{1}$, \\ Sylvio Klose ${ }^{2}$, and Thomas Krühler ${ }^{3}$ for the GROND team \\ ${ }^{1}$ Max-Planck-Institute für extraterrestrische Physik, \\ Giessenbachstraße 1, 85748, Garching, Germany, email: foe@mpe.mpg.de \\ ${ }^{2}$ Thüringer Landessternwarte Tautenburg, Sternwarte 5, 07778, Tautenburg, Germany \\ ${ }^{3}$ Dark Cosmology Centre, Niels Bohr Institute, University of Copenhagen, \\ Juliane Maries Vej 30, 2100, Copenhagen, Denmark
}

\begin{abstract}
We report on the type-Ic SN 2010bh associated with XRF 100316D at $z=0.059$, which is among the latest spectroscopically confirmed GRB-SNe (Bufano et al. 2012). This supernova proves to be the most rapidly evolving GRB-SN to date.
\end{abstract}

Keywords. supernovae: individual (SN 2010bh), gamma-rays: burts

\section{Introduction}

So far only a handful of supernovae (SNe) associated with gamma-ray bursts (GRBs) have been spectroscopically confirmed; see Woosley \& Bloom (2006) and Hjorth \& Bloom (2011) for reviews. Out of those only SN 2006aj associated with XRF 060218 has shown signatures of the cooling of the shock breakout (Campana et al. 2006).

For the case of the association SN 2010bh/XRF 100316D, GROND provided data from 0.5 to 80 days after the burst covering a wavelength range from 350 to $1800 \mathrm{~nm}$, significantly expanding the pre-existing data set for this event (e.g., Cano et al. 2011).

\section{Results}

Broad Band Spectral Energy Distribution: Detections at $50 \mathrm{ks}$ in $g^{\prime} r^{\prime} i^{\prime} z^{\prime} J$ are combined with a UVOT uvw1-band detection at $33 \mathrm{ks}$ and an interpolated XRT spectrum. We model the SED (left panel of Fig. 1) with a blackbody associated with the cooling of the shock breakout and a power-law representing the afterglow. We obtain a host-galaxy extinction of $A_{V \text {, host }}=1.2 \pm 0.1 \mathrm{mag}$ and a metal absorption equivalent to a hydrogen column density of $N_{H, \text { host }}=(4.4 \pm 0.4) \times 10^{22} \mathrm{~cm}^{-2}$.

Evolution of the Thermal Component: Early X-ray measurements from Starling et al. (2011) complement our results for the temperature and radius of the blackbody component (Fig. 1, right panel). Assuming a linear growth, we obtain $v=8000 \mathrm{~km} / \mathrm{s}$. The best model is a power law, which yields an initial apparent radius of $R_{0}=(7.0 \pm 0.9) \times$ $10^{11} \mathrm{~cm}$. As seen in the upper right panel of Fig. 1, adiabatic expansion fails to reproduce $T_{B B}(t)$.

Pseudo Bolometric Light Curve: The flux integrated from the $g^{\prime}$ to the $H$ bands was fitted using the two component model from Maeda et al. (2003), which consists of a dense inner core and an outer core with lower opacity (e.g., Valenti et al. 2008). The transition from optically thick to thin occurs at $\approx 33$ days after the burst for SN $2010 \mathrm{bh}$. The physical parameters of the explosion are $M_{\mathrm{Ni}}=(0.21 \pm 0.03) M_{\odot}, M_{\mathrm{ej}}=$ $(2.60 \pm 0.23) M_{\odot}$, and $E_{\mathrm{k}}=(2.4 \pm 0.7) \times 10^{52} \mathrm{erg}$. 

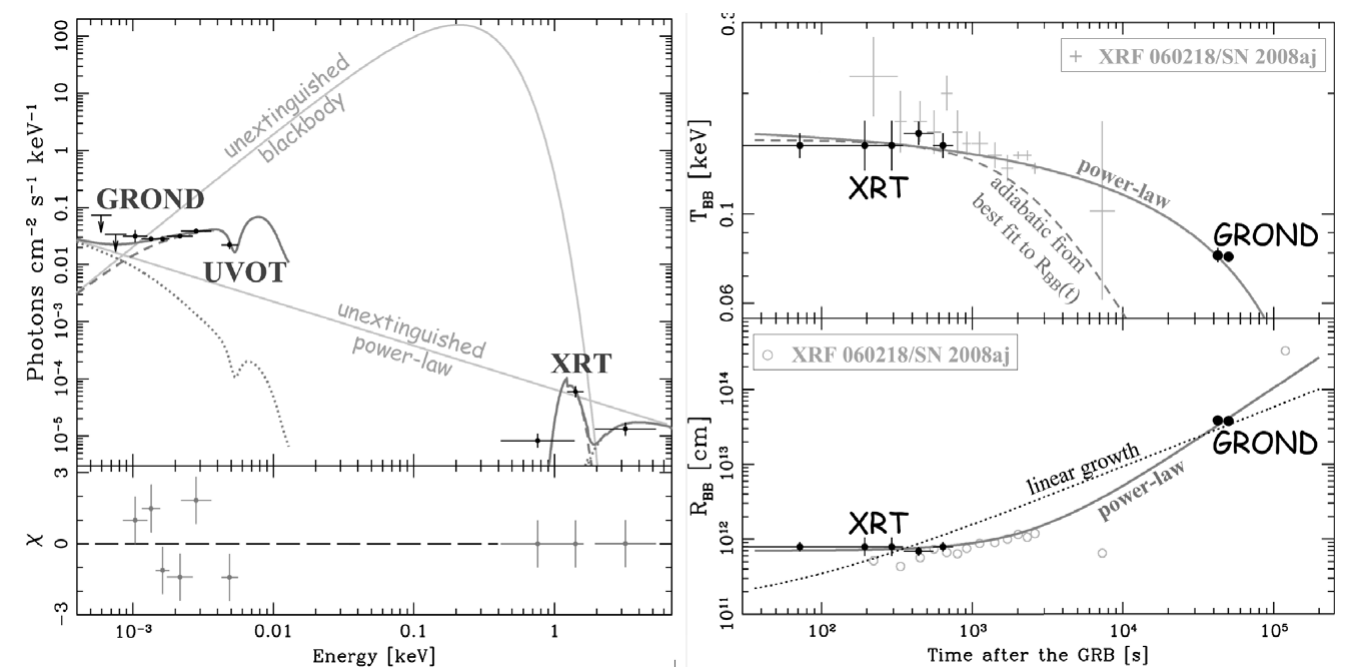

Figure 1. Left: Broad-band spectral energy distribution. The afterglow spectral slope is $\Gamma=1.8 \pm 0.1$. Right: Temporal evolution of the blackbody component. Temperature is shown in the upper panel and blackbody radius in the lower panel.

\section{Conclusions}

Combining GROND and Swift data, the early broad-band SED is modeled with a blackbody and power-law component attenuated by ISM absorption in the host galaxy. The evolution of the thermal component reveals a cooling envelope at an apparent initial radius, which is compatible with a dense wind surrounding a WR star. Moreover, the early-time expansion velocities are compatible with the SN nature. Multicolor templates of SN 1998bw show that SN 2010bh is on average $70 \%$ as bright as SN 1998bw (see Olivares E. et al. 2012). Reaching maximum brightness at 8-9 days after the burst in the blue bands, SN 2010bh is the most rapidly evolving GRB-SNe to date. A twocomponent parametrized model fitted to the pseudo bolometric light curve delivered physical parameters of the explosion. The kinetic energy makes this SN the second most energetic GRB-SN after SN 1998bw. SN 2010bh also shows one of the earliest peaks ever recorded and it fades more rapidly than any other GRB-SN or type-Ic SN. Further analysis can be found in Olivares E. et al. (2012).

\section{Acknowledgements}

Part of the funding for GROND (both hardware as well as personnel) was generously granted from the Leibniz-Prize to Prof. G. Hasinger (DFG grant HA 1850/28-1). This work made use of data supplied by the UK Swift Science Data Centre at the University of Leicester.

\section{References}

Bufano, F., Pian, E., Sollerman, J., et al. 2012, ApJ submitted, arXiv:1111.4527

Campana, S., Mangano, V., Blustin, A. J., et al. 2006, Nature, 442, 1008

Cano, Z., Bersier, D., Guidorzi, C., et al. 2011, ApJ, 740, 41

Hjorth, J. \& Bloom, J. S. 2011, in Gamma-Ray Bursts, ed. C. Kouveliotou, R. A. M. J. Wijers, \& S. E. Woosley (Cambridge: Cambridge Univ. Press), arXiv:1104.2274

Maeda, K., Mazzali, P. A., Deng, J., et al. 2003, ApJ, 593, 931

Olivares E., F., Greiner, J., Schady, P., et al. 2012, A\& $A$, 539, A76

Starling, R. L. C., Wiersema, K., Levan, A. J., et al. 2011, MNRAS, 411, 2792

Valenti, S., Benetti, S., Cappellaro, E., et al. 2008, MNRAS, 383, 1485

Woosley, S. E. \& Bloom, J. S. 2006, ARAA, 44, 507 